\title{
$\bar{\partial}$-Torsion for Complex Manifolds and the Adiabatic Limit
}

\section{Stephane Laederich}

Department of Mathematics University of Michigan, Ann Arbor, Michigan 48109-1003, USA

Received December 20, 1990; revised form September 16, 1991

Abstract. We consider a complex fibration $F \rightarrow M \stackrel{\pi}{\rightarrow} B$ and pull back bundles $E_{1}$ and $E_{2}$ over $M$. Using the adiabatic limit idea, we compute the metric invariant $T_{p}\left(E_{1}\right) / T_{p}\left(E_{2}\right)$, where $T_{p}(E)$ denotes the complex Ray-Singer torsion.

\section{Introduction}

In this paper we will study the Ray-Singer $\bar{\partial}$-torsion [RSI\&II] of complex manifolds which are fiber bundles. To be more specific, we consider the fibration

$$
\begin{gathered}
F \rightarrow M \\
\quad \downarrow \pi, \\
B
\end{gathered}
$$

where $F, B$ and $M$ are compact complex manifolds and where $\pi$ is holomorphically locally trivial. Given a hermitian bundle $\mathscr{E}$ over $B$, one wants to compute the $\bar{\partial}$-torsion $T_{p}\left(\pi^{*} \mathscr{E}\right)$ on $M$. We recall that the torsion is defined as follows:

$$
T_{p}\left(\pi^{*} \mathscr{E}\right)=\exp \left(\left.\frac{d}{d s}\right|_{s=0} \sum(-1)^{q} q \frac{1}{\Gamma(s)} \int_{0}^{\infty} t^{s-1} t r e^{-t \Delta^{p, q}} d t\right),
$$

where $\Delta^{p, q}$ is the $\bar{\partial}$-Laplacian on forms in $\Lambda^{p, q} T^{*} M \otimes \pi^{*} \mathscr{E}$ and where the right-hand side of (1.2) is to be understood as the derivative at $s=0$ of the analytic continuation of the sum. (For more details, we refer to [RSI] and Seeley [Se].) We will use the convention that $\Delta$ is a positive operator. Note also that (1.2) defines the $\bar{\partial}$-torsion to be the square of that of [RSI\&II]. In the case where the bundles are constructed by representations of $\pi_{1}(M), \mathrm{J}$. Fay [Fa], showed that (1.2) allowed to define analytic extensions of the torsion to non unitary representations.

One wishes to find formulas for the torsion in terms of the torsion of the base manifold. In the real case, such formulas are known. They can either be proved topologically (see D. Fried [Fr]) or analytically (see Forman [Fo] or Dai, Epstein and Melrose [DEM]). For acyclic pull-back bundles, these formulas are remark- 
ably simple: the torsion only depends on the torsion of the base on the bundles $\mathscr{E} \otimes H^{j} F$, where $H^{j} F$ is the $j^{\text {th }}$ cohomology bundle of the fiber over $B$. The analytical proofs of these formulas are based on the idea of adiabatic limits first introduced by Witten [Wi] and later used by Cheeger [C], Bismut and Cheeger [BC] and Dai [DI\&II] or [DEM], [Fo]. One chooses a metric on $M$ which makes the fibration (1.1) into a Riemannian submersion, that is

$$
g_{M}=\pi^{*} g_{B}+g_{F}
$$

One then introduces the modified metrics

$$
g_{M}^{\varepsilon}=\pi^{*} \varepsilon^{-2} g_{B}+g_{F}
$$

where $\varepsilon>0$. By taking the limit as $\varepsilon \rightarrow 0$ of the torsion and by using the metric invariance, it is possible to compute the torsion of the pull back bundle $\pi^{*} \mathscr{E}$.

Since we are interested in the complex case, for which the torsion is not (in general) a metric invariant (see [RSII]), we will consider two bundles $\mathscr{E}_{1}$ and $\mathscr{E}_{2}$ over the base $B$ and take ratios of torsions. We will require that

$$
\begin{aligned}
& \text { the bundles } \Lambda^{p_{1}, *-j} T^{*} B \otimes \mathscr{E}_{i} \otimes H^{p_{2}, j} \mathscr{F} \text { are acyclic } \\
& \text { for } i=1,2, p_{1}=0, \ldots, \operatorname{dim}_{\mathbb{C}} B \text { and } p_{2}, j=0, \ldots, \operatorname{dim}_{\mathbb{C}} F .
\end{aligned}
$$

That is, the Dolbeault complex with coefficients in $\mathscr{E}_{i} \otimes H^{p_{2}, j} F$ has no cohomology. Since these the cohomology of these bundles are the $E_{2}$ terms of the Leray-Hirsch spectral sequence of the fibration (see Bott \& Tu [BT] or Mazzeo-Melrose [MM]), the condition (1.4) implies that

$$
\Lambda^{p_{1}, *} T^{*} M \otimes \pi^{*} \mathscr{E}_{i}, \quad i=1,2 \text { are acyclic. }
$$

We have

Theorem 1.1. Under the condition (1.4), for $p=1, \ldots, \operatorname{dim}_{\mathbb{C}} M$, the limit as $\varepsilon \rightarrow 0$ of $\frac{T_{p}\left(\pi^{*} \mathscr{E}_{1}, \varepsilon\right)}{T_{p}\left(\pi^{*} \mathscr{E}_{2}, \varepsilon\right)}$ exists and is equal to

$$
\prod_{p_{1}+p_{2}=p} \prod_{j=0}^{\operatorname{dim} \sigma}\left[\frac{T_{p_{1}}\left(\mathscr{E}_{1} \otimes H^{p_{2}, j} F\right)}{T_{p_{1}}\left(\mathscr{E}_{2} \otimes H^{p_{2}, j} F\right)}\right]^{(-1)^{j}},
$$

where $T_{p}\left(\pi^{*} \mathscr{E}_{i}, \varepsilon\right)$ denotes the $\bar{\partial}$-torsion defined by $(1.2)$ for the $g_{M}^{\varepsilon}$ metric $(1.3 b)$ and where $T_{p}\left(\mathscr{E}_{i} \otimes H^{p_{2}, j} F\right)$ is the $\bar{\partial}$-torsion for the $g_{B}$ Laplacian on $B$ with coefficients in $\mathscr{E}_{i} \otimes H^{p_{2}, j} F$.

This formula differs from the one for real fibrations in two respects. First there is the product over $p$, which is a natural consequence of the splitting of $\Lambda^{p, q} T^{*} M \otimes \mathscr{E}$ in fiber and base components, and, more important, we have ratios of torsions. As showed in [RSII], this is a natural metric invariant object on compact complex manifolds.

Hence, we have

Corollary 1.2. For any Riemannian metric $g$ on $M$, under condition (1.4) and for any $p=0, \ldots, \operatorname{dim}_{\mathbb{C}} M$,

is given by (1.6).

$$
\frac{T_{p}\left(\pi^{*} \mathscr{E}_{1}\right)}{T_{p}\left(\pi^{*} \mathscr{E}_{2}\right)}
$$


The author has been made aware that in the case of locally Kähler fibrations, these results are implictly contained in the papers of Bismut, Gillet and Soule [BGS I, II \& III]. However, the techniques used here are based on different ideas and the proof is valid for arbitrary holomorphic fibrations.

A dynamical interpretation as well as examples of the use of such a product formula will be given in a subsequent paper. The idea is that on Kähler manifolds $M$ with a non-vanishing holomorphic vector field $v$, there is a flow-induced fibration over $B=\mathbb{C} / \mathbb{Z}+\tau \mathbb{Z}$ (see Crew and Fried $[\mathrm{CF}]$ ). The product formula then relates an invariant of the manifold to the dynamics on $M$, that is, as in the standard Lefschetz formula, counts closed orbits of the flow of $v$.

The rest of this paper is organized as follows: we first describe an extension of the $\bar{\partial}$-torsion which will allow us to simplify the proof of the theorem. We then analyse the spectrum of the $\Delta_{\varepsilon}^{p, q}$ Laplacian, that is, we investigate the asymptotically small eigenvalues of the Laplacian and we finish by computing their contribution to the trace of the heat kernel, that is to the torsion, as $\varepsilon \rightarrow 0$. It should be noted that it is this analysis which cannot be carried out in the more general case of a $C^{\infty}$ fibration of complex manifolds.

At this point, I would like to thank David Fried for having introduced me to this problem and for the constant support he provided during this work. I would also like to thank the many people who helped me during this work. To name but a few, they are: Xiangzhe Dai, Steve Rosenberg, Andrzej Lesniewski and Richard Melrose.

\section{The $\bar{\partial}$-Torsion}

We briefly define the $\bar{\partial}$-torsion ([RSI\&II]) and give a few of its properties. We borrow the notation of $\mathrm{F}$. Tangerman $[\mathrm{T}]$ in his announcement of the proof of the Ray-Singer conjecture using Witten's complex [Wi].

In the following we will let $E^{q}, q=0, \ldots, N$ be spaces of sections of bundles $\mathscr{E}^{q}$ over a fixed manifold $M$. We will denote the inner products on $E^{q}$ by $h^{g}$. Let $d^{q}$ be differential operators of degree $k$ (not necessarily equal to 1)

$$
d^{q}: E^{q} \rightarrow E^{q+1}
$$

such that $(E, h, d)$ is an elliptic complex. (See Atiyah-Bott [ABI\&II], Gilkey [Gi] or Wells [We]). Taking the adjoints $\left(d^{q}\right)^{*}$ with respect to $h^{q}$ of the operators $d^{q}$, we consider the corresponding Laplacians

$$
\Delta^{q}=\left(d^{q}\right)^{*} d^{q}+d^{q-1}\left(d^{q-1}\right)^{*}: E^{q} \rightarrow E^{q} .
$$

Following Seeley [Se], one can define a zeta function associated to the Laplacians.

Definition 2.1. For $s \in \mathbb{C}$ with $\operatorname{Re}(s)$ sufficiently large, define

$$
\zeta^{q}(s)=\frac{1}{\Gamma(s)} \int_{0}^{\infty} t^{s-1} \operatorname{tr}\left(e^{-t \Delta^{q}}-P_{q}\right) d t,
$$

where $P_{q}$ is the projection on $\operatorname{Ker} \Delta^{q}$.

In this paper, Seeley showed that $\zeta^{a}(s)$ is analytic for $\operatorname{Re}(s)$ sufficiently large and possesses an analytic extension which is regular at $s=0$. Using the zeta functions one can define a zeta-regularized determinant of $\Delta^{q}$ by 


\section{Definition 2.2.}

$$
\operatorname{det}_{\zeta} \Delta^{q}=\exp -\left.\frac{d}{d s}\right|_{s=0} \zeta^{q}(s) .
$$

We can now define the torsion of the elliptic complex $(E, h, d)$.

Definition 2.3. The torsion of the elliptic complex $(E, h, d)$ is defined by

$$
\operatorname{Tor}(E, h, d)=\prod_{q}\left(\operatorname{det}_{\zeta} \Delta^{q}\right)^{(-1)^{q+1} q}
$$

Note that $\operatorname{Tor}(E, h, d)$ will depend on the inner products $h^{q}$. It nevertheless has some nice properties which will be given.

Two cases will be of special interest. Let $M$ be a manifold and $E$ a flat bundle over $M$. Let $g$ be a metric on $M$. Choosing $E^{q}$ as the sections of $A^{q} T^{*} M \otimes E$ and letting $d^{q}$ be the usual exterior differentiation, $(E, g, d)$ is the de Rham complex and $\operatorname{Tor}(E, g, d)$ is the Reidemeister torsion. In the complex case, choosing $E^{q}$ to be sections over $\Lambda^{p, q} T^{*} M \otimes E$ and $d^{q}=\bar{\partial}$, that is, $(E, g, d)$ is the Dolbeault complex, $\operatorname{Tor}(E, g, d)$ is the $\bar{\partial}$-torsion $T_{p}(E)$. We now give some simple properties of the torsion.

Lemma 2.1. Let $\left(E_{1}, h_{1}, d_{1}\right)$ and $\left(E_{2}, h_{2}, d_{2}\right)$ be two elliptic complexes. Then

$$
\operatorname{Tor}\left(E_{1} \oplus E_{2}, h_{1} \oplus h_{2}, d_{1} \oplus d_{2}\right)=\operatorname{Tor}\left(E_{1}, h_{1}, d_{1}\right) \operatorname{Tor}\left(E_{2}, h_{2}, d_{2}\right) \text {. }
$$

The proof of this lemma is a straightforward application of the definition and will be omitted. For the next property, we need

Definition 2.4. The characteristic of the elliptic complex $(E, h, d)$ is defined as

$$
\chi(E)=\sum_{q=0}^{N}(-1)^{q} \operatorname{dim} H^{q}(E, d) .
$$

Given two complexes $\left(E_{1}, h_{1}, d_{1}\right)$ and $\left(E_{2}, h_{2}, d_{2}\right)$ with $d_{1}$ and $d_{2}$ of the some degree, one can build a third complex

$$
\tilde{E}^{q}=\bigoplus_{j=0}^{q} E_{1}^{q-j} \otimes E_{2}^{j}
$$

with the natural metric $\tilde{h}$ and differential operation $\tilde{d}$. One has

Lemma 2.2.

$$
\operatorname{Tor}(\tilde{E}, \tilde{h}, \tilde{d})=\operatorname{Tor}\left(E_{1}, h_{1}, d_{1}\right)^{\chi\left(E_{2}\right)} \operatorname{Tor}\left(E_{2}, h_{2}, d_{2}\right)^{\chi\left(E_{1}\right)}
$$

The proof of Lemma 2.2 is exactly the same as the proof of Theorem 3.3 of [RS II]. It is based on the fact that the heat kernel on $\widetilde{E}$ is given by

$$
k_{\tilde{E}}^{q}(t)=\sum_{j \leqq q} k_{E_{1}}^{q-j}(t) \otimes k_{E_{2}}^{j}(t)
$$

and on a theorem of T. Kotake [Ko] which gives the characteristic of a complex in terms of heat kernels:

$$
\chi(E)=\sum_{q}(-1)^{q} \operatorname{tre}^{-t \Delta q}
$$


When $\left(E_{1}, h_{1}, d_{1}\right)$ is acyclic, i.e. has no cohomology, one can rephrase Lemma 2.2 in terms of the bundles

$$
\mathscr{H}_{j}^{q}=E_{1}^{q} \otimes H^{j} E_{2},
$$

where $H^{j} E_{2}$ is the cohomology bundle of $E_{2}$. One has

Lemma 2.3. Let $\tilde{E}$ be as in (2.7). Then

$$
\operatorname{Tor}(\tilde{E}, \tilde{h}, \tilde{d})=\Pi_{j} \operatorname{Tor}\left(\mathscr{H}_{j}, h_{j}, d_{j}\right)^{(-1)^{j}} .
$$

Several other properties of the torsion do exist but will not be discussed here.

\section{The Splitting of the Torsion}

In order to simplify the notations we will let $E$ be either of the bundles $\pi^{*} \mathscr{E}_{i}, i=1,2$ and write $\Lambda^{p, q}(M, E)$ for $\Lambda^{p, q} T^{*} M \otimes E$. In many cases, we will drop the index $p$.

The main idea is to make a spectral decomposition of $\Lambda(M, E), i=1,2$ in terms of the behaviour of the eigenvalues of $\Delta_{\varepsilon}$ as $\varepsilon \rightarrow 0$. By using the properties of Tor(.,.,.), one can then study the torsion on each factor. The decomposition is done as follows:

Definition 3.1. For $\varepsilon>0$ and for $q=0, \ldots, \operatorname{dim}_{\mathbb{C}} M$ define

$$
\begin{aligned}
& \mathscr{P}^{p, q}(\varepsilon)=\left\{\omega \in \Lambda^{p, q}(M, E) \mid \Delta_{\varepsilon} \omega=\lambda(\varepsilon) \omega \text { and } \lambda(\varepsilon) \underset{\varepsilon \rightarrow 0}{\longrightarrow} 0\right\}, \\
& \mathscr{R}^{p, q}(\varepsilon)=\left\{\omega \in \Lambda^{p, q}(M, E) \mid \Delta_{\varepsilon} \omega=\lambda(\varepsilon) \omega \text { and } \min (\lambda(\varepsilon))>0\right\}
\end{aligned}
$$

and define

$$
S^{p, q}(\varepsilon)=\operatorname{span} \mathscr{S}^{p, q}(\varepsilon), \quad R^{p, q}(\varepsilon)=\operatorname{span} \mathscr{R}^{p, q}(\varepsilon)
$$

Due to the properties of $\Delta_{\varepsilon}^{q}$ one immediately obtains

Lemma 3.1. (i) $\Lambda^{q}(M, E)=S^{q}(\varepsilon) \oplus R^{q}(\varepsilon)$.

(ii) $\Delta_{\varepsilon}^{q}$ is diagonal with respect to this splitting of $\Lambda^{q}(M, E)$. By the results of the previous section, the torsion splits (for fixed $p$ ) as

$$
\operatorname{Tor}\left(\Lambda(M, E), g_{\varepsilon}, d_{\varepsilon}\right)=\operatorname{Tor}\left(S(\varepsilon), g_{\varepsilon}, d_{\varepsilon}\right) \operatorname{Tor}\left(R(\varepsilon), g_{\varepsilon}, d_{\varepsilon}\right) \text {. }
$$

In the subsequent sections, the limit as $\varepsilon \rightarrow 0$ of each of these factors will be discussed. Consider the bundles over $B$

$$
\bigoplus_{p_{1}+p_{2}=p} \bigoplus_{j} \Lambda^{p_{1}, q-j} T^{*} B \otimes \mathscr{E} \otimes H^{p_{2}, j} F
$$

and denote by $\Delta_{B}^{p, q}$ the $g_{B}$ Laplacian on these bundles. The following result, due to X. Dai, (see [D], Theorem 1.1), characterises the asymptotically small eigenvalues of $\Delta_{\varepsilon}$ in terms of the eigenvalues of $\Delta_{B}^{p, q}$.

Theorem 3.2. There exists a constant $\lambda_{0}$ such that for any eigenvalue $\lambda(\varepsilon)$ of $\Delta_{\varepsilon}^{p, q}$ on $R(\varepsilon)$,

$$
\lambda(\varepsilon) \geqq \lambda_{0}
$$


The eigenvalues of $\Delta_{\varepsilon}^{p, q}$ on $S(\varepsilon)$ have the asymptotics

$$
\lambda(\varepsilon)=\mu \varepsilon^{2}\left(1+c_{1}(\varepsilon) \mu \varepsilon\right)
$$

where the $\mu$ 's are the eigenvalues of $\Delta_{B}^{p, q}$ and where $c_{1}$ is uniformly bounded. The correspondence is between $\lambda$ 's and $\mu$ 's $1-1$ and there is a uniform constant $c_{2}$ such that $\left|\lambda(\varepsilon) \varepsilon^{-2}-\mu\right|<c_{2}$.

$X$. Dai proved this theorem for spin manifolds and Dirac operators. However, the proof carries over verbatim to the case of $D^{2}$ where $D=\vec{\partial}+\bar{\partial}^{*}$. Locally, $D$ is Dirac which is the only requirement for the proof to work. This theorem will allow us to compute the $S(\varepsilon)$ torsion directly. The $R(\varepsilon)$ torsion is a bit more complicated to compute. This will be done in the next section.

\section{The Large Eigenvalues Contribution: The $R(\varepsilon)$ Torsion}

We can now compute the contribution of the "large" eigenvalues of $\Delta_{\varepsilon}$ to the torsion as $\varepsilon \rightarrow 0$. That is, the contribution of $R(\varepsilon)$ as $\varepsilon \rightarrow 0$.

As defined in (3.2), we will denote by $R_{1}(\varepsilon), R_{2}(\varepsilon)$ the large eigenvalue part for each of the bundles $\mathscr{E}_{1}, \mathscr{E}_{2}$, the corresponding Laplacians by $\Delta_{1, \varepsilon}, \Delta_{2, \varepsilon}$, the Hodge star operators on $R_{i}(\varepsilon)$ by $\bar{x}_{i}$ and the adjoints of $d_{\varepsilon}$ by $\delta_{i, \varepsilon}$. We will also omit the superscripts $p, q$. We have

Theorem 4.1. $\lim _{\varepsilon \rightarrow 0} \frac{\operatorname{Tor}\left(R_{1}(\varepsilon), g_{\varepsilon}, d_{\varepsilon}\right)}{\operatorname{Tor}\left(R_{2}(\varepsilon), g_{\varepsilon}, d_{\varepsilon}\right)}=1$.

To prove this theorem, we will first investigate the small time behaviour of the heat kernels. Then, using the finite propagation speed techniques discussed in Cheeger, Gromov and Taylor [CGT], we estimate the contribution of the trace of the heat kernels to the torsion for $t$ bounded away from 0 .

4.1. The Small Time Behaviour. To analyse the small time behaviour, we make use of the well known asymptotics (see Ray-Singer [RSI \& II] for small times, stating that

$$
\operatorname{tr} e^{-t \Delta_{1}}-\operatorname{tr} e^{-t \Delta_{2}}=0\left(e^{-c / t}\right) \quad \text { as } \quad t \rightarrow 0 .
$$

Even though the proof of this formula is well known, we will give a short sketch in order to obtain careful estimates of the constants in terms of $\varepsilon$. We have

Proposition 4.2. For $t<\varepsilon^{-2}$, there exist constants $c_{1}$ and $c_{2}$, independent of $\varepsilon$ such that

$$
\left|\operatorname{tr} e^{-t \Delta_{1, \varepsilon}}-\operatorname{tr} e^{-t \Delta_{2, \mathrm{~b}}}\right| \leqq c_{1} e^{-c_{2} / \varepsilon^{2} t} .
$$

Proof. The proof is based on Duhamel's principle. We denote by $k_{i, \varepsilon}(t, x, y), i=1,2$ the kernels of $\Delta_{i, \varepsilon}$. We can then write for any simple connected $W \subset M, x, y \in W$,

$$
\begin{aligned}
\bar{*}_{2, y}^{-1} \bar{*}_{1, y} k_{1, \varepsilon}(t, x, y)-k_{2, \varepsilon}(t, x, y)= & \int_{0}^{t} d s \int_{W}\left[\Delta_{1, \varepsilon} k_{1, \varepsilon}(s, x, u) \wedge \bar{*}_{1} k_{2, \varepsilon}(t-s, u, y)\right. \\
& \left.-k_{1, \varepsilon}(s, x, u) \wedge \bar{*}_{1} \Delta_{2, \varepsilon} k_{2, \varepsilon}(t-s, u, y)\right] .
\end{aligned}
$$


Let $U=\left\{U_{\alpha}\right\}$ be a trivializing cover for the bundles $\mathscr{E}_{i}$. Without loss of generality we can assume that there are points $x_{z}$ in $B$ and a constant $c$ such that

$$
U_{\alpha}=\left\{x \in B / \rho_{\varepsilon}^{2}\left(x, x_{\alpha}\right) \leqq \frac{c}{\varepsilon^{2}}\right\} .
$$

This is a crucial fact. The bundles which one considers, that is $E_{i}=\pi^{*} \mathscr{E}_{i}, i=1,2$, are pull back bundles; whence (4.3). We define

$$
W_{\alpha}=U_{\alpha} \times F \text {. }
$$

The Laplacians $\Delta_{i, \varepsilon}, i=1,2$ are now equal on sections over $U_{\alpha}$. Hence as in [RSI] we can apply Green's formula to (4.2) to obtain

$$
\begin{aligned}
& k_{1, \varepsilon}(t, x, y)-k_{2, \varepsilon}(t, x, y) \\
& =-\int_{0}^{t} d s \int_{\partial W_{x}}\left[k_{2, \varepsilon}(t-s, u, y) \wedge \bar{*} d k_{1, \varepsilon}(x, u, s)-\delta k_{1, \varepsilon}(s, x, u) \wedge \bar{*} k_{2, \varepsilon}(t-s, u, y)\right. \\
& \left.\quad-k_{1, \varepsilon}(s, x, u) \wedge \bar{*} d k_{2, \varepsilon}(t-s, u, y)+\delta k_{1, \varepsilon}(t-s, u, y) \wedge \bar{*} k_{2, \varepsilon}(s, x, u)\right] .
\end{aligned}
$$

Now for $\varepsilon$ sufficiently small, $\partial W_{\alpha}=\left\{\rho_{\varepsilon}^{2}\left(x, x_{\alpha}\right)=\frac{c}{\varepsilon^{2}}\right\}$, so that we can use the standard
estimate

$$
\left|\nabla_{x}^{i_{1}} \nabla_{y}^{i_{2}} k(t, x, y)\right| \leqq c\left(i_{1}, i_{2}\right) e^{-\rho_{t}^{2}(x, y) / 4 t}
$$

for $\rho_{\varepsilon}(x, y)$ sufficiently large; (see [CGT] or Cheeger [C]). This estimate is obtained via finite propagation speed techniques (see [CGT]). Splitting the heat kernel into a finite range part, $\left(\rho_{\varepsilon}(x, y) \leqq b\right)$ and a remainder, one estimates each part separately. Choosing $b$ sufficiently large yields the above estimate. Using (4.6), we thus obtain the proposition.

4.2. The Median and Large Time Behaviour. We now turn our attention to the behaviour of the trace for $t_{0} \leqq t<\infty$. This will be done by the methods used in [CGT]. Let $\varphi(x)$ be an even $C^{\infty}$ function such that

$$
\begin{aligned}
& \operatorname{supp} \varphi \subset\left\{|x| \geqq \frac{\sqrt{\lambda_{0}}}{2}\right\}, \\
& \operatorname{supp}(1-\varphi) \subset\left\{|x| \leqq \sqrt{\lambda_{0}}\right\},
\end{aligned}
$$

and define

$$
H(x)=\varphi(x) e^{-x^{2}} .
$$

Now by Theorem 3.2, we have that

$$
\left|\operatorname{tr} e^{-t \Delta_{\varepsilon} \mid R(\varepsilon)}\right| \leqq\left|\operatorname{tr} H\left(\sqrt{\Delta_{\varepsilon}}\right)\right| .
$$

We will estimate the trace of $H\left(\sqrt{\Delta_{\varepsilon}}\right)$ via finite propagation speed techniques. We first remark that on $M$, the injectivity radius for the $g_{\varepsilon}$ is bounded away from zero and that the sectional curvature is bounded from above. Hence by (1.29) of [CGT] one has that

$$
\left|k_{H}\right| \leqq C_{1} \sum_{k=0}^{n+1} \int_{0}^{\infty}\left|\hat{H}^{(k)}(\xi)\right| d \xi,
$$


where $k_{H}$ denotes the Schwartzian kernel of $H\left(\sqrt{\Delta_{\varepsilon}}\right)$, where $n=\operatorname{dim}_{\mathbb{R}} M$, and where $\hat{H}^{(k)}$ denotes the $k^{\text {th }}$ derivative of the Fourier transform. As in Dai [D], we can estimate the Fourier transforms of $H$ by

$$
\begin{aligned}
\left|\hat{H}^{(k)}(\xi)\right| & \leqq \frac{2 C_{2}(k)}{t^{(k-1) / 2}} \int_{\xi \geqq \sqrt{\lambda_{0} / 2}} \xi e^{-t \xi^{2}} d \xi \\
& \leqq \frac{C_{2}(k)}{t^{(k-1) / 2}} e^{-t \lambda_{0} / 4} .
\end{aligned}
$$

To estimate the integral in (4.10) we write

$$
\int_{0}^{\infty}\left|\hat{H}^{k}(\xi)\right| d \xi=\int_{0}^{\infty}\left(1+\xi^{2}\right)\left|\hat{H}^{(k)}(\xi)\right| \frac{d \xi}{1+\xi^{2}}
$$

and

$$
\left(1+\xi^{2}\right) \hat{H}^{(k)}(\xi)=\hat{H}^{(k)}(\xi)+\left(\hat{H}^{\prime \prime}\right)^{(k)}(\xi)-2 k\left(\hat{H}^{\prime}\right)^{(k-1)}(\xi)-(k-1)^{2} \hat{H}^{(k-1)}(\xi)
$$

(cf. [D]). Hence, we obtain for some constants $N$ and $C_{3}$

$$
\left|k_{H}\right| \leqq \frac{C_{3}}{t^{N}} e^{-\lambda_{0} t / 4}
$$

and thus, by integration

$$
\left|\operatorname{tr} H\left(\sqrt{\Delta_{\varepsilon}}\right)\right| \leqq \frac{C_{4}}{\varepsilon^{p} t^{N}} e^{-t \lambda_{0} / 4}
$$

where $p=\operatorname{dim}_{\mathbb{R}} B$. Summarizing, we obtain

Lemma 4.3. There exist constants $N$ and $C$, independent of $\varepsilon$ such that

$$
\left|\operatorname{tr} e^{-t \Delta_{\varepsilon} \mid R(\varepsilon)}\right| \leqq \frac{C}{\varepsilon^{p} t^{N}} e^{-t \lambda_{0} / 4}
$$

where $p=\operatorname{dim}_{\mathbb{R}} B$.

4.3. Proof of Theorem 4.1. We can now prove Theorem 4.1. By the definition of Tor, we have to estimate

$$
\frac{1}{\Gamma(s)} \int_{0}^{\infty} t^{s-1}\left(\operatorname{tr} e^{-t \Delta_{1, \epsilon} \mid R(\varepsilon)}-\operatorname{tr} e^{-t \Delta_{2, \varepsilon} \mid R(\varepsilon)}\right) d t
$$

as $\varepsilon \rightarrow 0$. For $|s| \leqq \frac{1}{2}$, we have by Proposition 4.2 that

$$
\left|\frac{1}{\Gamma(s)} \int_{0}^{t_{0}} t^{s-1}\left[\operatorname{tr} e^{-t \Delta_{1, c} \mid R(\varepsilon)}-\operatorname{tr} e^{-t \Delta_{2, \varepsilon} \mid R(\varepsilon)}\right] d t\right| \leqq c \int_{0}^{t_{0}} t^{-3 / 2} e^{-c_{2} / \varepsilon^{2} t} d t
$$

for $t_{0}<\varepsilon^{-2}$.

Hence, choosing $t_{0}=\varepsilon^{-1}$, we have the uniform estimate of (4.15) by $C \varepsilon$ for any $s \in\left\{|s| \leqq \frac{1}{2}\right\}$. Similarly, using Lemma 4.3 , we have the estimate

$$
\left|\frac{1}{\Gamma(s)} \int_{t_{0}}^{\infty} t^{s-1} \operatorname{tr} e^{-t \Delta_{i, r}} d t\right| \leqq c \varepsilon^{-p} t_{0}^{-N-1 / 2} e^{-t_{0} \lambda_{0} / 4}, \quad i=1,2
$$

and $|s| \leqq \frac{1}{2}$ 
By our choice of $t_{0}$, the right-hand side of (4.16) can be estimated by $C \varepsilon$ for some constant $C$. Summing up these estimates we see that (4.14) is uniformly estimated by $3 C \varepsilon$ for $s \in\left\{|s| \leqq \frac{1}{2}\right\}$. Hence, the claim of the theorem.

\section{The Small Eigenvalue Contribution: The $S(\varepsilon)$ Torsion}

Using Theorem 3.2, it will be possible to compute the $S(\varepsilon)$ contribution to the torsion as $\varepsilon \rightarrow 0$. Before this is done, we briefly recall that the bundles

$$
\bigoplus_{p_{1}+p_{2}=p} \bigoplus_{j} \Lambda^{p_{1}, q-j} T^{*} B \otimes \mathscr{E}_{i} \otimes H^{p_{2}, j} F
$$

form an acyclic complex for $\bar{\partial}_{B}$ for $i=1,2$. Note that this operator increases $q$ by one. We denote by $\Delta_{i}^{p, q}$ the $\bar{\partial}$-Laplacian on the bundles defined by (5.1) and let

$$
\zeta_{\mathscr{E}_{i}}^{p, q}(s, \varepsilon)=\frac{1}{\Gamma(s)} \int_{0}^{\infty} t^{s-1} \operatorname{tr} e^{-\varepsilon^{2} t \Delta_{i}^{p, q}} d t .
$$

We also define the zeta functions on $S_{i}(\varepsilon)$ by

$$
\zeta_{i}^{p, q}(s, \varepsilon)=\frac{1}{\Gamma(s)} \int_{0}^{\infty} t^{s-1} \operatorname{tr} e^{-\Delta_{\varepsilon}^{p, q} \mid S_{i}(\varepsilon)} d t .
$$

We have

Lemma 5.1. Under the condition of acyclicity of (5.1)

$$
\left(\zeta_{\mathscr{E} 1}^{p, q}(s, \varepsilon)-\zeta_{\mathscr{E} 2}^{p, q}(s, \varepsilon)\right)
$$

and its first $s$-derivative are independent of $\varepsilon$ at $s=0$ for $p, q=0, \ldots, \operatorname{dim}_{\mathbb{C}} B$.

Proof. The proof is based on the fact that

$$
\frac{d}{d \varepsilon} \operatorname{tr} e^{-\varepsilon^{2} t \Delta^{p, q}}=t \frac{2}{\varepsilon} \frac{d}{d t} \operatorname{tr} e^{-\varepsilon^{2} t \Delta^{p, q}} .
$$

Using Proposition 4.2 and denoting by $j(s, \varepsilon)$ the difference $\zeta_{\mathscr{E}_{1}}^{p, q}(s, \varepsilon)-\zeta_{\mathscr{E}_{2}}^{p, q}(s, \varepsilon)$ we see that

$$
\frac{\partial}{\partial \varepsilon} j(s, \varepsilon)=\frac{2}{\varepsilon} \frac{1}{\Gamma(s)} \int_{0}^{\infty} t^{s} \frac{d}{d t} \operatorname{tr}\left(e^{-\varepsilon^{2} t \Delta_{1}^{p, q}}-e^{-\varepsilon^{2} t \Delta_{2}^{p, q}}\right) d t,
$$

and that the integrand is absolutely integrable. Hence, carrying out the integration, one obtains, since the bundles are acyclic,

$$
\frac{\partial}{\partial \varepsilon} j(s, \varepsilon)=0,\left.\quad \frac{\partial}{\partial \varepsilon} \frac{\partial}{\partial s} j(s, \varepsilon)\right|_{s=0}=0
$$

Thus $\left.\frac{\partial}{\partial s} j(s, \varepsilon)\right|_{s=0}$ is independent of $\varepsilon$.

We now denote by $\lambda_{i}(\varepsilon)$ the eigenvalues of $\Delta_{\varepsilon}^{p, q}$ on $S_{i}(\varepsilon), i=1,2$ and by $\mu_{i}$ the eigenvalues of $\Delta_{B}$ on the bundles (5.1). Using Proposition 4.2 on the bundles $\mathscr{E}_{i}, i=1,2$, we have 
Lemma 5.2. For $|s|<1 / 2$, for $t_{0}=\varepsilon^{-3 / 2}$, and for $p=0, \ldots, \operatorname{dim}_{\mathbb{C}} M, q=0, \ldots, \operatorname{dim}_{\mathbb{C}} M$,

and

$$
\left|\frac{1}{\Gamma(s)} \int_{0}^{t_{0}} t^{s-1} \sum_{\lambda_{1}(\varepsilon), \lambda_{2}(\varepsilon)}\left[e^{-\lambda_{1}(\varepsilon) t}-e^{-\lambda_{1}(\varepsilon) t}\right] d t\right| \leqq C \varepsilon
$$

$$
\left|\frac{1}{\Gamma(s)} \int_{0}^{t_{0}} t^{s-1} \sum_{\mu_{1}, \mu_{2}}\left[e^{-\mu_{1} \varepsilon^{2} t}-e^{-\mu_{2} \varepsilon^{2} t}\right] d t\right| \leqq C \varepsilon
$$

We have

$$
\zeta_{i}^{p, q}(s, \varepsilon)-\zeta_{\mathscr{E}_{i}}^{p, q}(s, \varepsilon)=\frac{1}{\Gamma(s)} \int_{0}^{\infty} t^{s-1} \sum_{\lambda_{i}(\varepsilon), \mu_{i}}\left[e^{-\lambda_{i}(\varepsilon) t}-e^{-\mu_{i} e^{2} t}\right] d t .
$$

By Lemmas 5.1 and 5.2, we only need to compute the integral on the right-hand side of (5.5) between $t_{0}=\varepsilon^{-3 / 2}$ and $\infty$. We now break the sum in the integral in (5.5) in a sum where $\lambda_{i} \geqq K \varepsilon$ and a sum where $\lambda_{i}<K \varepsilon$ for some constant $K$. These two sums and their contribution to (5.5) will be computed separately.

5.1. Breaking-up the Trace. By Theorem 3.2, to each $\lambda_{i}(\varepsilon)$ there corresponds a unique $\mu_{i}$ such that

$$
\lambda_{i}(\varepsilon)=\mu_{i} \varepsilon^{2}\left(1+c_{1}(\varepsilon) \mu_{i} \varepsilon\right)
$$

where $c_{1}(\varepsilon)$ is uniformly bounded. Hence, for any small enough constant $K$, if $\lambda_{i}(\varepsilon) \geqq$ $2 K \varepsilon$, the corresponding $\mu_{i} \geqq K / \varepsilon$. To see this, assume that for every $\varepsilon, \mu_{i}<K / \varepsilon$. Using (5.6), one immediately obtains that $\lambda_{i}(\varepsilon)<2 K \varepsilon$. For a small enough constant $\beta$, we see that if $\mu_{i}<\beta / \varepsilon$ then

$$
\mu_{i} \varepsilon^{2}<\lambda_{i}(\varepsilon)<\frac{3}{2} \beta
$$

Furthermore, since $\left|\varepsilon^{-2} \lambda_{i}(\varepsilon)-\mu_{i}\right|<c_{2}$, if $\lambda_{i}(\varepsilon)<2 K \varepsilon$, then $\mu_{i}<K / \varepsilon$. We now choose $K<\beta / 2$ and write

$$
\begin{aligned}
\sum_{\lambda_{i}(\varepsilon), \mu_{i}}\left[e^{-\lambda_{i}(\varepsilon) t}-e^{-\mu_{i} \varepsilon^{2} t}\right]= & \sum_{\lambda_{i}<2 K \varepsilon}\left[e^{-\lambda_{i}(\varepsilon) t}-e^{-\mu_{i} \varepsilon^{2} t}\right] \\
& +\sum_{\lambda_{i} \geqq 2 K \varepsilon}\left[e^{-\lambda_{i}(\varepsilon) t}-e^{-\mu_{i} \varepsilon^{2} t}\right] .
\end{aligned}
$$

In the first sum on the right-hand side of $(5.7)$, the $\mu_{i} \geqq K / \varepsilon$. The first sum on the right-hand side of (5.7) can be estimated by

$$
\left|\sum_{\mu_{i}<\beta / \varepsilon}\left[e^{-\lambda_{i}(\varepsilon) t}-e^{-\mu_{i} \varepsilon^{2} t}\right]\right| .
$$

5.2. Contribution to the Torsion. For the second sum on the right-hand side of (5.6), we use the same techniques as in the proof of Lemma 4.3. By choosing a function $H(x)$ as in (4.8), where $\lambda_{0}=2 K \varepsilon,(4.13)$ yields that for $i=1,2$,

$$
\sum_{\lambda_{i}(\varepsilon) \geqq 2 K \varepsilon}\left[e^{-\lambda_{i}(\varepsilon) t}-e^{-\mu_{i} \varepsilon^{2} t}\right] \leqq \frac{2 C}{\varepsilon^{D} t^{N}} e^{-t K \varepsilon / 4} .
$$

Hence, we obtain that for $i=1,2$,

$$
\left|\frac{1}{\Gamma(s)} \int_{t_{0}}^{\infty} t^{s-1}\left(\sum_{\lambda_{i}, \mu_{i}} e^{-\lambda_{i}(\varepsilon) t}-e^{-\mu_{i} \varepsilon^{2} t}\right) d t\right| \leqq 2 C \varepsilon^{-p}\left|\int_{t_{0}}^{\infty} t^{-N+s-1} e^{-(K \varepsilon / 4) t} d t\right|,
$$


where the sums runs over $\lambda_{i}(\varepsilon) \geqq 2 K \varepsilon$ and the corresponding $\mu_{i}$ 's. By our choice of $t_{0}=\varepsilon^{-3 / 2}$, for $|s|<\delta$ sufficiently small, we can thus estimate the right-hand side of (5.10) by $C \varepsilon$ for some constant $C$. We now estimate (5.8) by

$$
\sum e^{-\mu_{i} e^{2} t}\left|e^{-t\left(\mu_{i} \varepsilon^{2}-\lambda_{i}(\varepsilon)\right)}-1\right|,
$$

where the sum runs over $\mu_{i}<\beta / \varepsilon$. We now use the fact that when $\mu_{i}<\beta / \varepsilon, \lambda_{i}(\varepsilon)>\mu_{i} \varepsilon^{2} / 2$. Hence,

$$
\left|\mu_{i} \varepsilon^{2}-\lambda_{i}(\varepsilon)\right|<\frac{\mu_{i} \varepsilon^{2}}{2} .
$$

Since $\left|e^{-x}-1\right| \leqq|x| e^{-|x|}$ we can therefore estimate (5.11) by

$$
\sum_{\mu_{i}<\beta / \varepsilon} c_{1} \mu_{i}^{2} \varepsilon^{3} t e^{-\mu_{i} t / 2} .
$$

We therefore have

$$
\left|\frac{1}{\Gamma(s)} \int_{t_{0}}^{\infty} t^{s-1} \sum_{\mu_{i}<\beta / \varepsilon} \mu_{i}^{2} \varepsilon^{3} t e^{\mu_{i} \varepsilon^{2} t / 2} d t\right| \leqq \frac{C}{\varepsilon|\Gamma(s)|}\left|\int_{t_{0}}^{\infty} t^{s-2} \sum_{\mu_{i}<\beta / \varepsilon} e^{-\mu_{i} \varepsilon^{2} t / 4} d t\right|,
$$

where for this last step, we used the fact that $x^{2} e^{-\alpha x}$ takes its maximum value $4 \alpha^{2} e^{-2}$ at $x=2 \alpha^{-1}$. Hence, that $\mu_{i}^{2} t \exp \left(-\mu_{i} \varepsilon^{2} t / 4\right)$ is estimated by $C \varepsilon^{-1} t^{-1}$ for some constant $C$. Rescaling time by a factor $4 \varepsilon^{-2}$ in the integral on the right-hand side of (5.13), we estimate (5.13) by

$$
C \mid \varepsilon^{1-s \zeta_{\tilde{E}_{i}}^{p, q}(s-1,1) \mid .}
$$

By Seeley, [Se] or Shubin [Sh], this zeta function is analytic near $s=0$, and thus, for $|s|<\delta$ small enough, we can estimate (5.13) by $C_{\varepsilon^{1 / 2}}$. Summarising the estimates obtained in (5.4), (5.9) and (5.14), we see that for $|s|<\delta$ small enough, one has

$$
\left|\left[\zeta_{1}^{p, q}(s, \varepsilon)-\zeta_{\sigma_{1}}^{p, q}(s, \varepsilon)\right]-\left[\zeta_{2}^{p, q}(s, \varepsilon)-\zeta_{\varepsilon_{2}}^{p, q}(s, \varepsilon)\right]\right|<C \varepsilon^{1 / 2}
$$

for some constant $C$. Since the estimate is uniform in $s$ we can take derivatives $s=0$ of

$$
\left[\zeta_{1}^{p, q}(s, \varepsilon)-\zeta_{\varepsilon_{1}}^{p, q}(s, \varepsilon)\right]-\left[\zeta_{2}^{p, q}(s, \varepsilon)-\zeta_{E_{2}}^{p, q}(s, \varepsilon)\right] .
$$

Exponentiating the result and using Lemma 5.1, we see that

$$
\lim _{\varepsilon \rightarrow 0} \frac{\operatorname{Tor}\left(S_{1}(\varepsilon), g_{\varepsilon}, d_{\varepsilon}\right)}{\operatorname{Tor}\left(S_{2}(\varepsilon), g_{\varepsilon}, d_{\varepsilon}\right)}
$$

exists and that the limit is equal to

$$
\frac{\operatorname{Tor}\left(\underset{p_{1}+p_{2}=p}{\oplus} \bigoplus_{j} \Lambda^{p_{1}, q-j} T^{*} B \otimes \mathscr{E}_{1} \otimes H^{p_{2}, j} F, g_{B}, d_{B}\right)}{\operatorname{Tor}\left(\underset{p_{1}+p_{2}=p}{\oplus} \oplus_{j} \Lambda^{p_{1}, q-j} T^{*} B \otimes \mathscr{E}_{2} \otimes H^{p_{2}, j} F, g_{B}, d_{B}\right)},
$$

and hence using the properties of Tor derived in Sect. 2, that the limit is equal to (1.6) which concludes the proof of Theorem 1.1. 


\section{References}

ABI Atiyah, M., Bott, R.: A Lefschetz fixed point formula for elliptic complexes I. Ann. Math. 86, 374-407 (1967)

ABII Atiyah, M., Bott, R.: A Lefschetz fixed point formula for elliptic complexes II. Ann. Math. 88, 451-491 (1968)

BC Bismut, J. M., Cheeger, J.: $\eta$-invariants and their adiabatic limits. J. Am. Math. Soc. 2, 33-70 (1988)

BGSI Bismut, J. M., Gillet, H., Soulé, C.: Analytic torsion and holomorphic determinant bundles I, Bott Chern forms and analytic torsion. Commun. Math. Phys. 115, 49-78 (1988)

BGSII Bismut, J. M., Gillet, H., Soulé, C.: Analytic torsion and holomorphic determinant bundles II, Direct images and Bott Chern forms. Commun. Math. Phys. 115, 79-126 (1988)

BGSIII Bismut, J. M., Gillet, H., Soulé, C.: Analytic torsion and holomorphic determinant bundles III, Quillen metrics on holomorphic determinants. Commun. Math. Phys. 115, 301-351 (1988)

BT Bott, R., Tu, L.: Differential forms in algebraic topology. GTM, 82, Berlin, Heidelberg, New York: Springer 1986

BV Bismut, J. M., Vasserot, E.: The asymptotics of the Ray-Singer analytic torsion associated with high powers of a positive line bundle. Commun. Math. Phys. 115, 355-367 (1989)

C Cheeger, J.: $\eta$-invariants, the adiabatic approximation and conical singularities. J. Diff. Geom. 26, 175-221 (1987)

CGT Cheeger, J., Gromov, M., Taylor, M.: Finite propagation speed, kernel estimates for functions of the Laplace Operator and the geometry of complete Riemanian manifolds. J. Diff. Geom. 17, 15-53 (1982)

CF Crew, R., Fried, D.: Nonsingular holomorphic flows. Top. 25, (4) 471-473 (1986)

DI Dai, X.: Adiabatic limits non-multiplicativity of signature and the Leray spectral sequence. MIT Preprint (1990)

DII Dai, X.: Geometric invariants and their adiabatic limits, MIT Preprint (1990)

FA Fay, J.: Analytic torsion and Prym differentials. Ann. Math. Studies. Princeton, NJ: Princeton University Press 1981

DEM Dai, X., Epstein, C., Melrose, R.: Adiabatic limit of the Ray-Singer analytic torsion, in preparation

Fo Forman, R.: Personal communication

Fr Fried, D.: Lefschetz formulas for flows. Contemp. Math. 58 III, 19-69 (1987)

$G$ Gilkey, P.: Invariant theory, the heat equation and the Atiyah Singer index theorem. Publish or Perish 11, (1984)

Ko Kotake, T.: The fixed point theorem of Atiyah Bott via parabolic operators. Commun. Pure Appl. Math. XXII, 789-806 (1969)

MM Mazzeo, R., Melrose, R.: The adiabatic limit, Hodge Cohomology and Leray's spectral sequence for a fibration. J. Diff. Geom. 31, 185-213 (1990)

RSI Ray, D., Singer, 1.: R-torsion and the Laplacian on Riemannian Manifolds. Adv. Math. 7, 145-210 (1971)

RSII Ray, D., Singer, I.: Analytic torsion for complex manifolds. Ann. Math. 108, 1-39 (1978)

Se Seeley, R. T.: Complex powers of an Elliptic Operator, Proc. Symp. Pure Math. 10, 288-307 (1967)

Sh Shubin, M. A.: Pseudodifferential operators and spectral theory. Berlin, Heidelberg, New York: Springer 1987

T Tangerman, F.: Reidemeister torsion and analytic torsion: Announcement of a proof of the Ray Singer conjecture using the Witten Complex, Courant Institute (1989)

We Wells, R. O.: Differential analysis on complex manifolds. GTM vol. 65. Berlin, Heidelberg, New York: Springer 1986

Wi Witten, E.: Supersymmetry and Morse theory. Adv. Math. 4, 109-126 (1970) 